\title{
Reversible cerebral vasoconstriction syndrome after intercontinental airplane travel
}

\author{
Sabrina Anticoli ${ }^{1} \cdot$ Maria Cristina Bravi $^{1}$ (D) Francesca Romana Pezzella $^{1}$
}

Received: 15 February 2017/ Accepted: 29 March 2017/Published online: 5 April 2017

(C) SIMI 2017

A 51-year-old man was admitted to our Stroke Unit because of confusion, sleepiness, and weakness of the right limbs a few days after a recurrent thunderclap occipital headaches occurred for the first time during the descent phase of an intercontinental airplane flight lasting 12-h. He had no history of prior illness, and he did not take any medications or illicit drugs. He also denied any history of migraine. Clinical examination revealed normal blood pressure, normal heart rate and rhythm, and normal oxygenation. Emesis, photosensitivity, nystagmus, right upper and lower limb hemiparesis were noted, and the patient was uncooperative during the clinical examination. He reported having a recurrent severe headache occurring since the air travel. No hemorrhagic or ischemic lesions were seen on the admission head CT scan. Brain MRI-DWI showed multifocal restricted diffusion areas in the right cerebellar hemisphere, subcortical white matter of the centrum semiovale, knee and splenius of the corpus callosum, and anterior thalamus of the left hemisphere. An MRA demonstrated multiple narrowings of the basilar artery, posterior cerebral arteries and intracranial tract of the vertebral arteries. Similarly, we found multifocal segmental vasoconstrictions of the left internal carotid, right internal carotid and middle cerebral arteries (M1 tract) (Fig. 1a, c). Routine laboratory examinations revealed the presence of increased PCR values $(1.83 \mathrm{mg} /$ dl) and high ESR $(50 \mathrm{~mm})$. Chest X-ray study, echocardiography and ultrasound examinations of the arteries of

Maria Cristina Bravi

mcbravi@tiscali.it

1 Stroke Unit Emergency Department, S.Camillo-Forlanini Hospital, P.za C. Forlanini n.1, 00151 Rome, Italy the neck were unremarkable. The patient was started on intravenous nimodipine, methylprednisolone and analgesic treatment for headaches, and after 11 days was discharged free of the signs or symptoms presented on admission. During follow-up, the patient underwent a new MRI-DWI and MRA that showed, after 20 days from acute event, complete regression of the multifocal segmental vasoconstriction of the intracerebral arteries (Fig. 1b, d). Reversible cerebral vasoconstriction syndrome (RCVS) is characterized by a reversible and multifocal vasoconstriction of cerebral arteries and severe headaches with or without focal neurological deficits or seizures [1]. Thunderclap headache is the most common clinical feature together with nausea, vomiting, confusion and blurred vision while subarachnoid, intracerebral hemorrhage or ischemic stroke represents possible major complications. The pathogenesis remains poorly understood, but an alteration of cerebral vascular tone that occurs spontaneously or is triggered by endogenous/exogenous substances seems to be plausible [2]. Putative precipitants of RCVS are the postpartum state, vasoactive drugs, immunosuppressants or blood products, catecholamine-secreting tumors, cervical and cerebral largeartery lesions, and head trauma. Recently, a case of RCVS precipitated by airplane descent has been reported [3] The diagnosis of RCVS is based on vascular imaging (CT, MRI or digital angiography), and the abnormalities are usually multiple and bilateral resulting in severe narrowing of intracranial arteries (classical "sausage on a string") [1] Treatment of RCVS consists of calcium channel blockers (nimodipine or verapamil), glucocorticoids and analgesics for headaches. To our knowledge this is the second case of RCVS associated with air flight. It suggests that RCVS may be precipitated by air travel, and that milder forms of RCVS occurring after flying may 
Fig. 1 Magnetic resonance angiography findings (a, c) demonstrating a multifocal narrowing of intracerebral artery (black arrows) completely resolved after 20 days from the acute event (b, d)
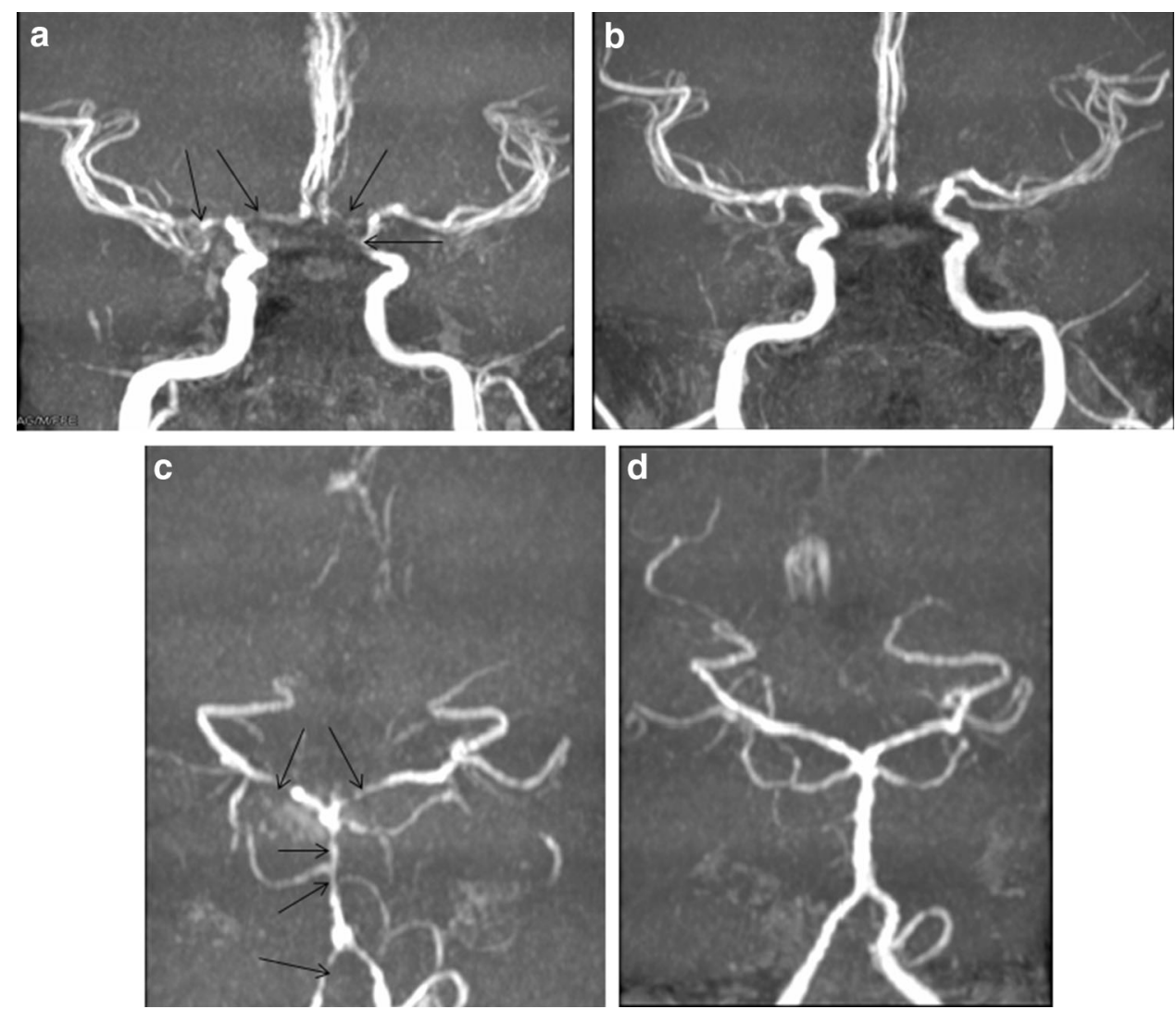

have previously been misdiagnosed and attributed to airplane travel headache or other form of cephalalgia.

\section{Compliance with ethical standards}

Conflict of interest The authors declare that they have no conflict of interest.

Statement of human and animal rights All procedures performed in this study were in accordance with the ethical standards of the institutional and/or national research committee.

\section{References}

1. Singhal AB, Hajj-Ali RA, Topcuoglu MA, Fok J, Bena J, Yang D, Calabrese LH (2011) Reversible cerebral vasoconstriction syndromes. Arch Neurol 68(8):1005-1012

2. Calabrese LH, Dodick DW, Schwedt TJ, Singhal AB (2007) Narrative review: reversible cerebral vasoconstriction syndromes. Ann Intern Med 146:34-44

3. Hiraga A, Aotsuka Y, Koide K, Kuwabara S (2016) Reversible cerebral vasocontriction syndrome precipitated by airplane descent: case report. Cephalalgia. doi:10.1177/0333102416665226

Informed consent Informed consent was obtained from patient. 